\title{
RIGIDITY OF INVARIANT COMPLEX STRUCTURES
}

\author{
ISABEL DOTTI MIATELLO
}

\begin{abstract}
A Kähler solvmanifold is a connected Kähler manifold $(M, j,\langle\rangle$, admitting a transitive solvable group of automorphisms. In this paper we study the isomorphism classes of Kähler structures $(j,\langle\rangle$,$) turning M$ into a Kähler solvmanifold. In the case when $(M, j,\langle\rangle$,$) is irreducible and simply$ connected we show that any Kähler structure on $M$, having the same group of automorphisms, is isomorphic to $(j,\langle\rangle$,$) .$
\end{abstract}

\section{INTRODUCTION}

A Kähler solvmanifold is a connected Kähler manifold $(M, j,\langle\rangle$,$) which$ admits a transitive solvable group $R$ of automorphisms (= holomorphic isometries). Two Kähler solvmanifolds $(M, j,\langle\rangle$,$) and (\widetilde{M}, \tilde{j},\langle\langle\rangle\rangle$,$) will be$ considered isomorphic if there exists a biholomorphic map $\varphi:(M, j,\langle\rangle,) \rightarrow$ $(\widetilde{M}, \tilde{j},\langle\langle\rangle\rangle$,$) . Since the pullback, by a diffeomorphism, of a Kähler structure$ is again a Kähler structure we will often say that two pairs $(j,\langle\rangle),,(\tilde{j},\langle\langle\rangle\rangle$, (on a fixed $M$ ) are isomorphic if the corresponding solvmanifolds $(M, j,\langle$,$\rangle )$ and $(M, \tilde{j},\langle\langle\rangle\rangle$,$) are isomorphic.$

The problem we will consider in this paper is related to the number of isomorphism classes of Kähler structures $(j,\langle\rangle$,$) on M$ turning it into a Kähler solvmanifold.

There are two basic types of Kähler solvmanifolds: (i) locally flat homogeneous Kähler manifolds $\left(C^{n} \times T^{k}\right.$ as $C^{\lambda}$ manifolds), (ii) homogeneous bounded domains. Moreover, it was proved by Dorfmeister in [4] that every Kähler solvmanifold fibers over a homogeneous bounded domain and each fiber is a locally flat Kähler manifold. Concerning the question we posed above it is known: (i) in $C^{n}$ all invariant complex structures are equivalent and in $T^{k}$ there exist nontrivial deformations of invariant complex structures; (ii) irreducible homogeneous bounded domain have, up to conjugation, a unique isomorphism class of Kähler structure with a fixed group of automorphisms [5].

It is well known (see [7]) that every Kähler solvmanifold $(M, j,\langle\rangle$,$) ad-$ mits an almost simply transitive solvable group of automorphisms. Thus we may identify $(M, j,\langle\rangle$,$) with (R / \Gamma, j,\langle\rangle$,$) where R$ is a simply-connected solvable group, $j$ is an invariant complex structure, $\langle$,$\rangle is a left invariant$

Received by the editors March 18, 1991.

1980 Mathematics Subject Classification (1985 Revision). Primary 17B05, 32M10, 32G05.

Research was partially supported by Conicet-Conicor Argentina. Part of this work was done during a stay at ICTP, Trieste, Italy. 
Kähler metric and $\Gamma$ is a discrete subgroup of

$$
\{x \in R: \operatorname{Ad}(x) \text { is orthogonal and complex linear }\} \text {. }
$$

In particular, simply-connected Kähler solvmanifolds can be identified with solvable Kähler algebras (see $\S 2$ ) and irreducible and simply connected Kähler solvmanifolds can be identified with solvable Kähler algebras that cannot be decomposed into a sum of $j$ invariant ideals. We call such algebras irreducible.

The main purpose of this paper is to prove

Theorem 6.1. Let $(M, j,\langle\rangle$,$) be an irreducible Kähler solvmanifold with$ $M$ simply connected and $G$ the group of automorphisms of $M$. Then any other Kähler structure $(\tilde{j},\langle\langle\rangle\rangle$,$) on M$ with group of automorphism $G$ is isomorphic to $(j,\langle\rangle$,$) .$

The proof of the above theorem reduces to the case when $(\mathfrak{r}, j,\langle\rangle$,$) , the$ solvable Kähler algebra associated to $(M, j,\langle\rangle$,$) , satisfies that the eigenval-$ ues of $\operatorname{ad}_{x}$ are real numbers, that is $\mathfrak{r}$ is a split solvable Lie algebra. This is so, since solvable Kähler algebras are normal modifications of split solvable ones by a result of Dorfmeister (see [4]). For irreducible and split solvable Kähler algebras, with no restriction on the group of automorphisms of the corresponding solvmanifold, we prove that every Lie algebra isomorphism gives rise to a complex (up to conjugation) isomorphism (Corollary 5.1).

\section{BASIC NOTATION AND RESULTS}

A solvable Kähler algebra $(\mathfrak{r}, j,\langle\rangle$,$) is a solvable Lie algebra \mathfrak{r}$ together with an inner product $\langle$,$\rangle and an orthogonal map j: \mathfrak{r} \rightarrow \mathfrak{r}$ satisfying

$$
\begin{gathered}
j^{2}=-\mathrm{id}, \\
j[x, y]=[j x, y]+[x, j y]+j[j x, j y], \\
\langle[x, y], j z\rangle+\langle[y, z], j x\rangle+\langle[z, x], j y\rangle=0 .
\end{gathered}
$$

A solvable Kähler algebra is said to be irreducible if it cannot be decomposed into a sum of $j$-invariant ideals. A split solvable Kähler algebra is a solvable Kähler algebra such that the eigenvalues of $\operatorname{ad}_{x}, x \in \mathfrak{r}$, are real numbers. A normal $j$-algebra is a split solvable Kähler algebra such that $\langle x, y\rangle=\omega[j x, y]$, $x, y \in r$, where $\omega$ is a linear form on $\mathfrak{r}$. Normal $j$-algebras will be denoted $(\mathfrak{r}, j, \omega)$. We note that normal $j$-algebras do not possess $j$-invariant abelian ideals. Also, if $\phi(x, y)=-\langle x, j y\rangle$ is the Kähler form then (3) says that $\phi$ is closed. On the other hand normal $j$-algebras are the split solvable Kähler algebras whose Kähler form $\phi$ is exact.

Given a real vector space $V$ with an endomorphism $j$ satisfying $j^{2}=-$ id and a skew-symmetric bilinear form $\rho,(V, j, \rho)$ is a symplectic space if for any $u, v \in V$,

$$
\rho(j u, j v)=\rho(u, v), \quad \rho(j u, u)>0, \quad u \neq 0 .
$$

Let $\mathfrak{r}$ be a solvable Kähler algebra and let $u$ be an abelian $j$-invariant ideal. Then $\mathfrak{s}$, the orthogonal complement of $\mathfrak{u}$ is a $j$-invariant subalgebra (by using (3)) hence a solvable Kähler algebra and for every $x \in \mathfrak{s}$,

$$
j \operatorname{ad}_{x}-\operatorname{ad}_{j x}=\operatorname{ad}_{x} j+j \operatorname{ad}_{j x} j
$$


when restricted to $u$. Furthermore, $(u, j, \rho)$ is a symplectic space and for every $x \in \mathfrak{s}, \operatorname{ad}_{x}$ is a symplectic transformation of $\mathfrak{u}$. In fact, it follows from (3) that if $u, v \in \mathfrak{u}, x \in \mathfrak{r}$,

$$
\rho\left(\operatorname{ad}_{x} u, v\right)+\rho\left(u, \operatorname{ad}_{x} v\right)=\langle[x, u], j v\rangle+\langle[x, v], j u\rangle=0 .
$$

Conversely, let $(\mathfrak{u}, j, \rho)$ be a symplectic space, let $(\mathfrak{s}, j,\langle\rangle$,$) be a solvable$ Kähler algebra and let $\mu: \mathfrak{s} \rightarrow g l(\mathfrak{u})$ be a representation by symplectic transformations such that $\operatorname{ad}_{x} u=\mu(x)(u)$ satisfies (5). Then $u \oplus \mathfrak{s}$ inherits a structure of solvable Kähler algebra with $u$ as a $j$-invariant abelian ideal.

Moreover, it was proved by Gindikin, Vinberg and Pyatetskii-Shapiro that this construction is general, that is, every split solvable Lie algebra can be obtained as above. We state their theorem and some consequences. All proofs can be found in [6].

Theorem 2.1 (Gindikin, Vinberg, Pyatetskii-Shapiro). Every split solvable Kähler algebra $(\mathfrak{r}, j,\langle\rangle$,$) can be decomposed into a semidirect sum$

$$
\mathfrak{r}=\mathfrak{u} \oplus \mathfrak{s}
$$

where $\mathfrak{u}$ is a commutative $j$-invariant ideal and $\mathfrak{s}$ is a normal $j$-algebra. Every normal $j$-algebra has an element $s$ with the following properties:

1. $[j s, s]=s$,

2. the operator $\left.\operatorname{ad}_{j s}\right|_{\mathfrak{s}}$ is semisimple and has eigenvalues $0,1, \frac{1}{2}$ with eigenspaces $\mathfrak{s}_{0}, \mathfrak{s}_{1}, \mathfrak{s}_{\frac{1}{2}}$ respectively,

3. $j \mathfrak{s}_{0}=\mathfrak{s}_{1}, j \mathfrak{s}_{1}=\mathfrak{s}_{0}, j \mathfrak{s}_{\frac{1}{2}}=\mathfrak{s}_{\frac{1}{2}}$.

2.1 Some consequences. See [6] for the proofs of the statements below.

1. The operator $\left.\operatorname{ad}_{j s}\right|_{\mathfrak{u}}$ is semisimple and has eigenvalues $0, \pm \frac{1}{2}$ with eigenspaces $\mathfrak{u}_{0}, u_{ \pm \frac{1}{2}}$ satisfying $j \mathfrak{u}_{\lambda}=\mathfrak{u}_{-\lambda}, \lambda=0, \pm \frac{1}{2}$.

2. $\left[\mathfrak{u}_{0}, \mathfrak{s}_{1}\right]=0,\left[\mathfrak{u}_{0}, \mathfrak{s}_{0}\right]=0$.

3. The decomposition of

$$
\mathfrak{r}=\mathfrak{u}_{0} \oplus \mathfrak{u}_{\frac{1}{2}} \oplus \mathfrak{u}_{-\frac{1}{2}} \oplus \mathfrak{s}_{1} \oplus \mathfrak{s}_{0} \oplus \mathfrak{s}_{\frac{1}{2}}
$$

is orthogonal with respect to $\langle$,$\rangle .$

4. $\mathfrak{z}$, the center of $\mathfrak{r}$, is a $j$-invariant ideal.

2.2 Decomposition of normal $j$-algebras. Let $(\mathfrak{r}, j,\langle\rangle$,$) be a split solvable$ Kähler algebra with decomposition $\mathfrak{r}=\mathfrak{u} \oplus \mathfrak{s}$ (given by Theorem 2.1) with $\mathfrak{u}$ abelian and $\mathfrak{s}$ a normal $j$-algebra. According to the structure theorem of Pyatetskii-Shapiro [8], a normal $j$-algebra $(\mathfrak{s}, j, \omega)$ can be decomposed $\mathfrak{s}=$ $\mathfrak{a} \oplus \mathfrak{n}$ (an orthogonal direct sum of vector spaces) where $\mathfrak{a}$ is abelian and $\mathfrak{n}=$ $[\mathfrak{s}, \mathfrak{s}]$. Moreover (i) the subalgebra $\mathfrak{n}$ can be represented as an orthogonal direct sum of the root spaces $\mathfrak{n}_{\alpha}=\{x \in \mathfrak{n}:[h, x]=\alpha(h) x, h \in \mathfrak{a}\}$ with respect to the adjoint action of $\mathfrak{a}$ on $\mathfrak{n}$; (ii) there are roots $\varepsilon_{1}, \ldots, \varepsilon_{r}, r=\operatorname{dim} \mathfrak{a}$, such that $j \mathfrak{a} \subset \mathfrak{n}$ is the direct sum of the one dimensional root spaces $\mathfrak{n}_{\varepsilon_{i}}, i=1, \ldots, r$, and with proper labelling all other roots are of the form $\frac{1}{2} \varepsilon_{k}, \frac{1}{2}\left(\varepsilon_{k} \pm \varepsilon_{m}\right)$, $1 \leq k<m \leq r$ (although not all these need be roots). Also $j \mathfrak{n}_{\frac{1}{2} \varepsilon_{k}}=\mathfrak{n}_{\frac{1}{2} \varepsilon_{k}}$ and $j \mathfrak{n}_{\frac{1}{2}\left(\varepsilon_{k} \pm \varepsilon_{m}\right)}=\mathfrak{n}_{\frac{1}{2}\left(\varepsilon_{k} \mp \varepsilon_{m}\right)}$.

The following notation will be used frequently in this paper.

If $(\mathfrak{s}, j, \omega)$ is a normal $j$-algebra we will let $\mathfrak{a}$ denote the orthogonal complement of $\mathfrak{n}=[\mathfrak{s}, \mathfrak{s}]$ with respect to the inner product induced from $\omega$. Since 
$\varepsilon_{k}\left(j \underline{n}_{\varepsilon_{m}}\right)=0, m \neq k$ and $\varepsilon_{k}\left(j \underline{n}_{\varepsilon_{k}}\right) \neq 0$, (see [2]) we will fix $x_{k} \in \mathfrak{n}_{\varepsilon_{k}}$ satisfying $\varepsilon_{i}\left(j x_{k}\right)=\delta_{i k}$.

\section{COMPLEMENTARY RESUlTS}

Keeping the notation of the previous section we prove now a series of lemmas. First we note that given a split solvable Kähler algebra $\mathfrak{r}=\mathfrak{u} \oplus \mathfrak{s}$,

$$
\mathfrak{v} \subset \mathfrak{u} \text { for any abelian } j \text {-invariant ideal } \mathfrak{v} \text {. }
$$

In fact, given $v \in \mathfrak{v}$, write $v=v_{\mathfrak{u}}+v_{\mathfrak{s}}$ with respect to the decomposition given in Theorem 2.1. Now

$$
0=[j v, v]=\left[j v_{\mathfrak{u}}, v_{\mathfrak{s}}\right]+\left[j v_{\mathfrak{s}}, v_{\mathfrak{u}}\right]+\left[j v_{\mathfrak{s}}, v_{\mathfrak{s}}\right]
$$

implies $v_{\mathfrak{s}}=0\left(\right.$ since $\left.\left\langle v_{\mathfrak{s}}, v_{\mathfrak{s}}\right\rangle=\omega\left[j v_{\mathfrak{s}}, v_{\mathfrak{s}}\right]\right)$. In particular the decomposition of Theorem 2.1 is unique.

Lemma 3.1. If $(\mathfrak{r}, j,\langle\rangle$,$) is a split solvable Kähler algebra then j s \in \mathfrak{a}$ and $\mathfrak{u}_{0} \oplus \mathfrak{a}$ is an abelian subalgebra which coincides with its own centralizer.

Proof. We note first that $\mathfrak{a} \subset \mathfrak{s}_{0}$ since by Theorem 2.1, [js, $\left.\mathfrak{s}_{1} \oplus \mathfrak{s}_{\frac{1}{2}}\right]=\mathfrak{s}_{1} \oplus \mathfrak{s}_{\frac{1}{2}}$ and the decomposition $\mathfrak{s}_{1} \oplus \mathfrak{s}_{0} \oplus \mathfrak{s}_{\frac{1}{2}}$ is orthogonal. Because $\left[\mathfrak{u}_{0}, \mathfrak{s}_{0}\right]=0$ it follows that $\mathfrak{u}_{0} \oplus \mathfrak{a}$ is abelian.

We verify next that $s$ given in Theorem 2.1 satisfies $j s \in \mathfrak{a}$. It is clear that $j s \in \mathfrak{s}_{0}$ thus $s \in \mathfrak{s}$. Now

$$
\left\langle\left[x_{\alpha}, y_{\beta}\right], j s\right\rangle=-\left\langle\left[s, x_{\alpha}\right], j y_{\beta}\right\rangle-\left\langle\left[y_{\beta}, s\right], j x_{\alpha}\right\rangle=0
$$

since $\alpha+1$ and $\beta+1$ are not roots. To prove the last assertion let $u+x \in \mathfrak{u} \oplus \mathfrak{s}$ be an element centralizing $\mathfrak{u}_{0} \oplus \mathfrak{a}$. In particular $x$ centralizes $\mathfrak{a}$ and by the structure theory of normal $j$-algebras it follows that $x \in \mathfrak{a}$. Using that $j s \in \mathfrak{a}$ one obtains that $u \in u_{0}$ as claimed.

Lemma 3.2. If $(\mathfrak{r}, j,\langle\rangle$,$) is a split solvable Kähler algebra then \mathfrak{z} \oplus \mathfrak{a}$ is the orthogonal complement of $[\mathfrak{r}, \mathfrak{r}]$.

Proof. Clearly $\langle\mathfrak{z},[\mathfrak{r}, \mathfrak{r}]\rangle=\langle j \mathfrak{z},[\mathfrak{r}, \mathfrak{r}]\rangle=0$ (see 2.1 ), thus $\mathfrak{z} \oplus \mathfrak{a}$ is orthogonal to $[\mathfrak{r}, \mathfrak{r}]$. Conversely if $\langle j u+x,[\mathfrak{r}, \mathfrak{r}]\rangle=0, u \in \mathfrak{u}, x \in \mathfrak{s}$ then $u \in \mathfrak{u}_{0}$ and $x \in \mathfrak{a}$. Furthermore $\langle j u,[\mathfrak{u}, \mathfrak{r}]\rangle=0$ if and only if for every $v \in \mathfrak{u}$ and $x \in \mathfrak{r}$,

$$
0=\langle j u,[v, x]\rangle=\langle[u, x], j v\rangle
$$

or equivalently $u$ (hence $j u$ ) is in the center $\mathfrak{z}$.

We observed that, as a consequence of (6), the decomposition $\mathfrak{r}=\mathfrak{u} \oplus \mathfrak{s}$ of a split solvable Kähler algebra was unique. The following lemma shows in particular that, up to an element in the center, there exists a unique $s$ with the properties listed in Theorem 2.1 .

Lemma 3.3. Let $(\mathfrak{r}, j,\langle\rangle$,$) be a split solvable Kähler algebra and let \bar{s} \in \mathfrak{r}$, $\overline{\mathfrak{s}} \subset \mathfrak{r}$ satisfying

(j) $\overline{\mathfrak{s}}$ is a subalgebra of $\mathfrak{r}$ such that $\mathfrak{r}=\mathfrak{u} \oplus \overline{\mathfrak{s}}$ (not necessarily orthogonal).

(jj) $\left.\operatorname{ad}_{j \bar{s}}\right|_{\overline{\mathfrak{s}}}$ is semisimple with eigenvalues $0,1, \frac{1}{2}$. If $\overline{\mathfrak{s}}_{\lambda}, \lambda=0,1, \frac{1}{2}$, denotes the corresponding eigenspaces then $j \overline{\mathfrak{s}}_{0}=\overline{\mathfrak{s}}_{1}, j \overline{\mathfrak{s}}_{\frac{1}{2}}=\overline{\mathfrak{s}}_{\frac{1}{2}}$.

Then $\bar{s}-s \in \mathfrak{z}$ and $\overline{\mathfrak{s}}_{i}=\mathfrak{s}_{i}$.

Proof. We assume first $\bar{s} \in \overline{\mathfrak{s}}_{1}$. Since $\mathfrak{u}$ is $j$-invariant, $\mathfrak{r} / \mathfrak{u}$ inherits a structure of a normal $j$-algebra. Moreover, if $\pi: \mathfrak{r} \rightarrow \mathfrak{r} / \mathfrak{u}$ is the quotient map, then 
$\pi(s)$ and $\pi(\bar{s})$ satisfy (i), (ii) and (iii) of Theorem 2.1. It follows from [6, p. 41], that $\pi(s)=\pi(\bar{s})$ hence $\bar{s}=u+s, u \in \mathfrak{u}$. In particular $\left.\operatorname{ad}_{j \bar{s}}\right|_{\mathfrak{u}}=\left.\operatorname{ad}_{j s}\right|_{\mathfrak{u}}$. Since $[j \bar{s}, \bar{s}]=\bar{s}$ and $[j s, s]=s$ it follows that $[j u, s]+[j s, u]=u$. If $u=u_{0}+u_{\frac{1}{2}}+u_{-\frac{1}{2}}$ then it follows from 2.1 that $[j u, s]=\left[j u_{\frac{1}{2}}, s\right]$ and from (2) that $\left[j u_{\frac{1}{2}}, s\right]=u_{\frac{1}{2}}$. Thus $u=[j u, s]+[j s, u]=u_{\frac{1}{2}}+\frac{1}{2} u_{\frac{1}{2}}-\frac{1}{2} u_{-\frac{1}{2}}$ implies $u \in \mathfrak{u}_{0}$. On the other hand if $x \in \overline{\mathfrak{s}}$, and $u \in \mathfrak{u}_{0}$ then,

$$
0=\langle[j \bar{s}, x], j u\rangle+\langle[u, j \bar{s}], j x\rangle-\langle[x, u], \bar{s}\rangle=\langle x, j u\rangle-\langle[x, u], \bar{s}\rangle .
$$

Since $[j \bar{s}[x, u]]=[x, u]$ and 1 is not an eigenvalue of $\operatorname{ad}_{j s}$ in $u$ it follows that $\left\langle\overline{\mathfrak{s}}, \mathfrak{u}_{0}\right\rangle=0$ thus $u=0$ and $\bar{s}=s$.

If $\bar{s} \in \mathfrak{r}$ then $\bar{s}=v+\overline{\bar{s}}$ with $v \in \mathfrak{u}, \overline{\bar{s}} \in \overline{\mathfrak{s}}$. Since $\operatorname{ad}_{\bar{s}}: \overline{\mathfrak{s}} \rightarrow \overline{\mathfrak{s}}, \overline{\mathfrak{s}}$ is a subalgebra and $\mathfrak{u}$ is abelian it follows that $v \in \mathfrak{z}$. Moreover, if $j \bar{s}=a_{0}+a_{1}+a_{\frac{1}{2}}$ with respect to the decomposition $\overline{\mathfrak{s}}_{0} \oplus \overline{\mathfrak{s}}_{1} \oplus \overline{\mathfrak{s}}_{\frac{1}{2}}$ then $0=a_{1}+\frac{1}{2} a_{\frac{1}{2}}$. Thus $\overline{\bar{s}} \in \overline{\mathfrak{s}}_{1}$ and $\overline{\bar{s}}$ satisfies (j) and (jj) of the lemma.

The proof of $\overline{\mathfrak{s}}_{i}=\mathfrak{s}_{i}$ is immediate from the fact $\bar{s}-s \in \mathfrak{z}$.

Remark 1. It follows from (6) and Lemma 3.3 that if $(\mathfrak{r}, j,\langle\rangle),,(\mathfrak{r}, j,\langle\langle\rangle\rangle$, are split solvable Kähler algebras with decompositions

$$
\mathfrak{r}=\mathfrak{u} \oplus \mathfrak{s}=\overline{\mathfrak{u}} \oplus \overline{\mathfrak{s}}
$$

given by Theorem 2.1 then $\mathfrak{u}=\overline{\mathfrak{u}}$ and $\mathfrak{s}_{i}=\overline{\mathfrak{s}}_{i}$.

Remark 2. It was proved in [3] that the orthogonal complement of $[\mathfrak{r}, \mathfrak{r}]$ in a normal $j$-algebra does not depend on the metric. This fact together with the previous remark and

$$
\langle j \mathfrak{z},[\mathfrak{r}, \mathfrak{r}]\rangle=\langle\langle j \mathfrak{z},[\mathfrak{r}, \mathfrak{r}]\rangle\rangle
$$

imply that the same result holds for split solvable Kähler algebras.

3.1 Decomposition of split solvable Kähler algebras. The next proposition is a consequence of Theorem 2.1, the structure theorem of Pyatetskii-Shapiro and Lemmas 3.1 and 3.2.

Proposition 3.1. If $\mathfrak{r}=\mathfrak{u} \oplus \mathfrak{s}$ is a split solvable Kähler algebra and $\mathfrak{s}=\mathfrak{a} \oplus \mathfrak{n}$ is a normal $j$-algebra with fundamental set of roots $\Lambda=\left\{\varepsilon_{1}, \ldots, \varepsilon_{r}\right\}$ then

$$
\mathfrak{u}=\mathfrak{u}_{0} \oplus \sum_{i \in J} \mathfrak{u}_{ \pm \frac{1}{2} \varepsilon_{i}}
$$

where $J \subset\{1, \ldots, r\},\left[\mathfrak{a}, \mathfrak{u}_{0}\right]=0,\left[j x_{i}, u\right]= \pm \frac{1}{2} \delta_{i k} u, u \in \mathfrak{u}_{ \pm \frac{1}{2} \varepsilon_{k}}$ and $\left[x_{i}, u\right]=j u, u \in \mathfrak{u}_{-\frac{1}{2} \varepsilon_{i}}$. Furthermore, the previous decomposition satisfies $\left[\mathfrak{u}_{-\frac{1}{2} \varepsilon_{i}}, \mathfrak{n}_{\frac{1}{2} \varepsilon_{k}}\right]=0, i \neq k$, hence, $\mathfrak{u}_{0}$ decomposes orthogonally

$$
\mathfrak{u}_{0}=\mathfrak{z} \oplus \sum_{i \in J}\left[\mathfrak{u}_{-\frac{1}{2} \varepsilon_{i}}, \mathfrak{n}_{\frac{1}{2} \varepsilon_{i}}\right] .
$$

Proof. Set $\mathfrak{h}_{i}=\operatorname{span}\left\{j x_{i}, x_{i}\right\}, i=1, \ldots, r$. Assume $\operatorname{ad}_{j x_{i}}: \mathfrak{u} \rightarrow \mathfrak{u}$ is not trivial and let $\mathfrak{u}=\mathfrak{u}_{0}^{i} \oplus \mathfrak{u}_{\frac{1}{2}}^{i} \oplus \mathfrak{u}_{-\frac{1}{2}}^{i}$ be the decomposition of $\mathfrak{u}$ given by Theorem 2.1 when considering the split solvable Lie algebra $\mathfrak{u} \oplus \mathfrak{h}_{i}$. If $u \in \mathfrak{u}_{-\frac{1}{2}}^{i},\left[j x_{i}, u\right]=$ $-\frac{1}{2} u$ and the Jacobi identity imply $\left[x_{i}, u\right] \in \mathfrak{u}_{\frac{1}{2}}^{i}$ and $\left[x_{i}, j u\right]=0$. Thus, using (2) one obtains

$$
\left[x_{i}, u\right]=j u .
$$

Also, from $\S 2.1,\left[x_{i}, v\right]=0$ if $v \in u_{0}^{i}$. 
Let now $k \neq i$. Since $\left[j x_{k}, j x_{i}\right]=\left[x_{k}, j x_{i}\right]=0$, we may consider the split solvable Lie algebra $\mathfrak{u}_{\frac{1}{2}}^{i} \oplus \mathfrak{u}_{-\frac{1}{2}}^{i} \oplus \mathfrak{h}_{k}$ and apply again Theorem 2.1. If $x_{+}+x_{-} \in \mathfrak{u}_{\frac{1}{2}}^{i} \oplus \mathfrak{u}_{-\frac{1}{2}}^{i}$ is an eigenvector of $\operatorname{ad}_{j x_{k}}$ with eigenvalue $\frac{1}{2}$, since $\operatorname{ad}_{j x_{k}}$ preserves $\mathfrak{u}_{\frac{1}{2}}^{i}$ and $\mathfrak{u}_{-\frac{1}{2}}^{i}$ we have $\left[j x_{k}, x_{+}\right]=\frac{1}{2} x_{+}$and $\left[j x_{k}, x_{-}\right]=\frac{1}{2} x_{-}$. If $x_{+} \neq 0$, then 1 will be an eigenvalue of $\operatorname{ad}_{j\left(x_{i}+x_{k}\right)}$. We thus get a contradiction by considering the split solvable Kähler algebra $\mathfrak{u}_{\frac{1}{2}}^{i} \oplus \mathfrak{u}_{-\frac{1}{2}}^{i} \oplus \mathfrak{h}_{i, k}$ where

$$
\mathfrak{h}_{i, k}=\operatorname{span}\left\{j\left(x_{i}+x_{k}\right), x_{i}+x_{k}\right\} \text {. }
$$

Analogously, the eigenvectors of $\operatorname{ad}_{j x_{k}}$ with eigenvalue $-\frac{1}{2}$ are in $\mathfrak{u}_{\frac{1}{2}}^{i}$. Thus

$$
\mathfrak{u}_{\frac{1}{2}}^{i}=\mathfrak{u}_{-\frac{1}{2}}^{i k} \oplus \mathfrak{v}_{0}^{i k}, \quad \mathfrak{u}_{-\frac{1}{2}}^{i}=\mathfrak{u}_{\frac{1}{2}}^{i k} \oplus \mathfrak{m}_{0}^{i k}\left(\mathfrak{u}_{\frac{1}{2}}^{i k}=j \mathfrak{u}_{-\frac{1}{2}}^{i k}\right) .
$$

Let $u \in u_{-\frac{1}{2}}^{i k}$. By (7), $\left[x_{k}, u\right]=j u$ and $\left[x_{i}, j u\right]=-u$. Then

$$
0=\left[x_{i}\left[x_{k}, u\right]\right]+\left[x_{k}\left[u, x_{i}\right]\right]+\left[u\left[x_{i}, x_{k}\right]\right]=-u
$$

since $\left[x_{i}, x_{k}\right]=0$ and $\left[u, x_{i}\right]=0$. In particular we have proved that $\operatorname{ad}_{j x_{k}} \mid \mathfrak{u}_{\frac{1}{2}}^{i}$ $\oplus \mathfrak{u}_{-\frac{1}{2}}^{i}$ is trivial hence $\mathfrak{u}_{0}^{i}$ contains the $\pm \frac{1}{2}$ eigenspace of $\operatorname{ad}_{j x_{k}}$. By repeating this process we obtain the claimed decomposition where the set $J=\{i=$ $\left.1, \ldots, r: \operatorname{ad}_{j x_{i}} \neq 0\right\}$.

To prove the last assertion we first note that

$$
\left[\mathfrak{u}_{-\frac{1}{2} \varepsilon_{i}}, \mathfrak{n}_{\frac{1}{2} \varepsilon_{k}}\right] \subset\left[\mathfrak{u}_{-\frac{1}{2}}, \mathfrak{s}_{\frac{1}{2}}\right] \subset \mathfrak{u}_{0} .
$$

Thus, if $u \in \mathfrak{u}_{-\frac{1}{2} \varepsilon_{i}}, x \in \mathfrak{n}_{\frac{1}{2} \varepsilon_{k}},\left[j x_{k}[u, x]\right]=0$ since, by Lemma 3.1, [a, $\left.\mathfrak{u}_{0}\right]=$ 0 . On the other hand, by the Jacobi identity, if $i \neq k,\left[j x_{k}[u, x]\right]=\frac{1}{2}[u, x]$. Hence $\left[\mathfrak{u}_{-\frac{1}{2} \varepsilon_{i}}, \mathfrak{n}_{\frac{1}{2} \varepsilon_{k}}\right]=0$. In particular, if $x \in \mathfrak{n}_{\frac{1}{2} \varepsilon_{i}}, v \in \mathfrak{u}_{-\frac{1}{2} \varepsilon_{k}}, y \in \mathfrak{n}_{\frac{1}{2} \varepsilon_{k}}$, it follows that $[[v, y] x]=[y[x, v]]+[v[y, x]]=0$ thus $\langle[y, x], j[v, y]\rangle=$ $\langle[[v, y] x], j u\rangle=0$. But, using (2) one shows that $\left[\mathfrak{u}_{-\frac{1}{2} \varepsilon_{i}}, \mathfrak{n}_{\frac{1}{2} \varepsilon_{i}}\right]$ is $j$-invariant. Thus, the previous relation imply $\left\langle\left[\mathfrak{u}_{-\frac{1}{2} \varepsilon_{i}}, \mathfrak{n}_{\frac{1}{2} \varepsilon_{i}}\right],\left[\mathfrak{u}_{-\frac{1}{2} \varepsilon_{k}}, \mathfrak{n}_{\frac{1}{2} \varepsilon_{k}}\right]\right\rangle=0, i \neq k$. Finally, because of Lemma 3.2 it follows that the decomposition $\mathfrak{u}_{0}=\mathfrak{z} \oplus$ $\left[\mathfrak{u}_{\frac{1}{2}}, \mathfrak{s}_{\frac{1}{2}}\right]=\sum\left[\mathfrak{u}_{-\frac{1}{2} \varepsilon_{i}}, \mathfrak{n}_{\frac{1}{2} \varepsilon_{i}}\right]$ is orthogonal as claimed.

\section{VARYING THE COMPLEX STRUCTURES}

Assume now that there exist two pairs $(j,\langle\rangle$,$) and (\tilde{j},\langle\langle\rangle\rangle$,$) turning$ $(\mathfrak{r}, j,\langle\rangle$,$) and (\mathfrak{r}, \tilde{j},\langle\langle\rangle\rangle$,$) into split solvable Kähler algebras. Then,$ according to Theorem 2.1,

$$
\mathfrak{r}=\mathfrak{u} \oplus \mathfrak{s}=\tilde{\mathfrak{u}} \oplus \tilde{\mathfrak{s}}
$$

where $\mathfrak{u}$ (resp. $\tilde{\mathfrak{u}})$ is an abelian $j$-invariant (resp. $\tilde{j}$-invariant) ideal and $\mathfrak{s}$ (resp. $\tilde{\mathfrak{s}}$ ) is a normal $j$-algebra. Let a (resp. $\tilde{\mathfrak{a}}$ ) be the orthogonal complement of $[\mathfrak{s}, \mathfrak{s}]=\mathfrak{n}$ in $\mathfrak{s}(\operatorname{resp} .[\tilde{\mathfrak{s}}, \tilde{\mathfrak{s}}]=\tilde{\mathfrak{n}}$ in $\tilde{\mathfrak{s}})$. The main purpose of this section is to understand the relation between $\mathfrak{u}$ and $\tilde{\mathfrak{u}}, \mathfrak{s}$ and $\tilde{\mathfrak{s}}$. The next proposition shows that $\mathfrak{u}_{0}$ determines $\mathfrak{u}$. 
Proposition 4.1. Let $(\mathfrak{r}, j,\langle\rangle$,$) and (\mathfrak{r}, \tilde{j},\langle\langle\rangle\rangle$,$) be two split solvable Kähler$ algebras with $\mathfrak{u}_{0}=\tilde{\mathfrak{u}}_{0}$. Then $\mathfrak{u}=\tilde{\mathfrak{u}}$.

Proof. Decompose $\mathfrak{r}$, according to Theorem 2.1, into the eigenspaces of $\operatorname{ad}_{j s}$,

$$
\mathfrak{r}=\left(\mathfrak{u}_{0} \oplus \mathfrak{s}_{0}\right) \oplus \mathfrak{s}_{1} \oplus\left(\mathfrak{u}_{\frac{1}{2}} \oplus \mathfrak{s}_{\frac{1}{2}}\right) \oplus \mathfrak{u}_{-\frac{1}{2}}=\mathfrak{r}_{0} \oplus \mathfrak{r}_{1} \oplus \mathfrak{r}_{\frac{1}{2}} \oplus \mathfrak{r}_{-\frac{1}{2}}
$$

and let $\tilde{\mathfrak{u}}$ be a $\tilde{j}$-invariant abelian ideal. We will show that $\tilde{\mathfrak{u}} \subset \mathfrak{u}$. By reversing the roles of $j$ and $\tilde{j}$ the lemma will follow.

If $x \in \tilde{u}$, then with respect to the above decomposition

$$
\begin{aligned}
x=x_{0}+x_{1}+x_{\frac{1}{2}}+x_{-\frac{1}{2}}, & {[j s, x]=x_{1}+\frac{1}{2} x_{\frac{1}{2}}-\frac{1}{2} x_{-\frac{1}{2}}, } \\
{[j s[j s, x]]=x_{1}+\frac{1}{4} x_{\frac{1}{2}}+\frac{1}{4} x_{-\frac{1}{2}}, } & {[j s[j s[j s, x]]]=x_{1}+\frac{1}{8} x_{\frac{1}{2}}-\frac{1}{8} x_{-\frac{1}{2}} . }
\end{aligned}
$$

Hence

$$
\frac{1}{4} x_{\frac{1}{2}}-\frac{3}{4} x_{-\frac{1}{2}}=[j s, x]-[j s[j s, x]] \in \tilde{\mathfrak{u}}
$$

and

$$
\frac{1}{4} x_{\frac{1}{2}}+\frac{3}{4} x_{-\frac{1}{2}}=2([j s[j s, x]]-[j s[j s[j s, x]]]) \in \tilde{u} .
$$

It then follows that all of the components of $x$ are in $\tilde{\mathfrak{u}}$ or equivalently

$$
\tilde{\mathfrak{u}}=\tilde{\mathfrak{u}} \cap \mathfrak{r}_{0} \oplus \tilde{\mathfrak{u}} \cap \mathfrak{r}_{1} \oplus \tilde{\mathfrak{u}} \cap \mathfrak{r}_{\frac{1}{2}} \oplus \tilde{\mathfrak{u}} \cap \mathfrak{r}_{-\frac{1}{2}} .
$$

Let $x \in \tilde{\mathfrak{u}} \cap \mathfrak{r}_{1}$ and $y \in \tilde{\mathfrak{u}} \cap \mathfrak{r}_{\lambda}, \lambda \in\left\{0,1, \frac{1}{2},-\frac{1}{2}\right\}$. Then

$$
0=\langle\langle[j s, x], \tilde{j}\rangle\rangle+\langle\langle[y, j s], \tilde{j} x\rangle\rangle=\langle\langle x, \tilde{j} y\rangle\rangle-\lambda\langle\langle y, \tilde{j} x\rangle\rangle .
$$

Since $1+\lambda \neq 0$ it then follows that $\tilde{\mathfrak{u}} \cap \mathfrak{r}_{1}=0$.

Let us now consider $\tilde{\mathfrak{u}} \cap \mathfrak{r}_{\frac{1}{2}}=\tilde{\mathfrak{u}} \cap\left(\mathfrak{u}_{\frac{1}{2}} \oplus \mathfrak{s}_{\frac{1}{2}}\right)$. If $x \in \tilde{\mathfrak{u}} \cap \mathfrak{r}_{\frac{1}{2}}$, and $x=u+y$ with $u \in \mathfrak{u}_{\frac{1}{2}}$ and $y \in \mathfrak{s}_{\frac{1}{2}}$ then $j x=j u+j y \in \mathfrak{u}_{-\frac{1}{2}} \oplus \mathfrak{s}_{\frac{1}{2}}$ and $[x, j x]=$ $[u, j y]+[y, j u]+[y, j y]$ is in $\tilde{\mathfrak{u}}$. Now, since $[u, j y]=0,[y, j u] \in \mathfrak{u}_{0}=\tilde{\mathfrak{u}}_{0}$ it then follows $[y, j y] \in \tilde{\mathfrak{u}} \cap \mathfrak{r}_{1}=0$. From (3)

$$
\langle[y, j y], s\rangle=\langle[j s, y], j j y\rangle+\langle[j y, j s], j y\rangle=-\langle y, y\rangle
$$

one obtains $y=0$ hence $\tilde{\mathfrak{u}} \cap \mathfrak{r}_{\frac{1}{2}} \subset \mathfrak{u}_{\frac{1}{2}}$.

Finally we show that $\tilde{\mathfrak{u}} \cap \mathfrak{r}_{0} \subset \mathfrak{u}_{0}$. If $x \in \tilde{\mathfrak{u}} \cap \mathfrak{r}_{0}$ then $x=u+y, y \in \mathfrak{u}_{0}, y \in \mathfrak{s}_{0}$. As above, by considering $[x, j x]=[u, j y]+[y, j u]+[y, j y] \in \tilde{\mathfrak{u}}$ and using that $[u, j y]=[y, j u]=0($ see 2.1$)$ one thus obtains $[y, j y] \in \tilde{u} \cap \mathfrak{r}_{1}=0$. Arguing as above one shows that $y=0$ and the proposition follows. let

If $(\mathfrak{r}, j,\langle\rangle$,$) and (\mathfrak{r}, \tilde{j},\langle\langle\rangle\rangle$,$) are two split solvable Kähler algebras we$

respectively

$$
\mathfrak{u}=\mathfrak{u}_{0} \oplus \mathfrak{u}_{\frac{1}{2}} \oplus \mathfrak{u}_{-\frac{1}{2}}, \quad \mathfrak{s}=\mathfrak{s}_{0} \oplus \mathfrak{s}_{1} \oplus \mathfrak{s}_{\frac{1}{2}}
$$

$$
\tilde{\mathfrak{u}}=\tilde{\mathfrak{u}}_{0} \oplus \tilde{\mathfrak{u}}_{\frac{1}{2}} \oplus \tilde{\mathfrak{u}}_{-\frac{1}{2}}, \quad \tilde{\mathfrak{s}}=\tilde{\mathfrak{s}}_{0} \oplus \tilde{\mathfrak{s}}_{1} \oplus \tilde{\mathfrak{s}}_{\frac{1}{2}}
$$

be the decompositions of $\mathfrak{u}, \mathfrak{s}$ (respectively, $\tilde{\mathfrak{u}}, \tilde{\mathfrak{s}}$ ) into eigenspaces of $\operatorname{ad}_{j s}$ (respectively $\mathrm{ad}_{\tilde{j} \tilde{s}}$ ).

Proposition 4.2. Let $(\mathfrak{r}, j,\langle\rangle$,$) and (\mathfrak{r}, \tilde{j},\langle\langle\rangle\rangle$,$) be two split solvable Kähler$ algebras with $\mathfrak{u}_{0} \oplus \mathfrak{a}=\tilde{\mathfrak{u}}_{0} \oplus \tilde{\mathfrak{a}}$. Then

(i) $\mathfrak{u}_{0}=\tilde{\mathfrak{u}}_{0}$ (hence $\left.\mathfrak{u}=\tilde{\mathfrak{u}}\right)$, 
(ii) $\mathfrak{u}_{\frac{1}{2}} \oplus \mathfrak{u}_{-\frac{1}{2}}=\tilde{\mathfrak{u}}_{\frac{1}{2}} \oplus \tilde{\mathfrak{u}}_{-\frac{1}{2}}$, the orthogonal complement of $\mathfrak{u}_{0}$ in $\mathfrak{u}$ with respect to both metrics coincide,

(iii) $\tilde{j} \tilde{s} \in \mathfrak{z} \oplus \mathfrak{a}, j s \in \mathfrak{z} \oplus \tilde{\mathfrak{a}}$,

(iv) $\left\langle\tilde{\mathfrak{s}}_{1}, \mathfrak{u}\right\rangle=0$.

Proof. (i) Decompose orthogonally with respect to $\langle\rangle,, \mathfrak{u}_{0} \oplus \mathfrak{a}=\mathfrak{z} \oplus \mathfrak{v} \oplus \mathfrak{a}$ and with respect to $\langle\langle\rangle\rangle,, \tilde{\mathfrak{u}}_{0} \oplus \tilde{\mathfrak{a}}=\mathfrak{z} \oplus \tilde{\mathfrak{v}} \oplus \tilde{\mathfrak{a}}$. If $x \in \mathfrak{v}$ then $x=z+\tilde{v}+a$ with respect to the last decomposition, hence, since $x$ and $\tilde{v}$ are in $[\mathfrak{r}, \mathfrak{r}]$ one has $z+a=0$ thus $\mathfrak{v} \subset \tilde{\mathfrak{v}}$. Similarly $\tilde{\mathfrak{v}} \subset \mathfrak{v}$ hence $\mathfrak{u}_{0}=\mathfrak{z} \oplus \mathfrak{v}=\mathfrak{z} \oplus \tilde{\mathfrak{v}}=\tilde{\mathfrak{u}}_{0}$.

(ii) Decompose $\mathfrak{u}$ with respect to both metrics

$$
\mathfrak{u}=\mathfrak{u}_{0} \oplus \mathfrak{u}_{\frac{1}{2}} \oplus \mathfrak{u}_{-\frac{1}{2}}=\tilde{\mathfrak{u}}=\tilde{\mathfrak{u}}_{0} \oplus \tilde{\mathfrak{u}}_{\frac{1}{2}} \oplus \tilde{\mathfrak{u}}_{-\frac{1}{2}} .
$$

Let $x \in \mathfrak{u}_{ \pm \frac{1}{2}}$ and $y \in \mathfrak{u}_{0}$. From (3) and using the fact that $\mathfrak{u}$ is abelian one has

$$
0=\langle\langle[j s, x], \tilde{j} y\rangle\rangle+\langle\langle[y, j s], \tilde{j} x\rangle\rangle= \pm\langle\langle x, \tilde{j} y\rangle\rangle
$$

Now, since $\mathfrak{u}_{0}=\tilde{\mathfrak{u}}_{0}$ is $\tilde{j}$-invariant (ii) follows.

(iii) We note that $\mathfrak{v}$, the orthogonal complement of $\mathfrak{z}$ in $\mathfrak{u}_{0}$, coincides with $\left[\mathfrak{u}_{-\frac{1}{2}}, \mathfrak{s}_{\frac{1}{2}}\right]$. By (i) above $\mathfrak{v}=\left[\tilde{\mathfrak{u}}_{-\frac{1}{2}}, \tilde{\mathfrak{s}}_{\frac{1}{2}}\right]$ thus if $u \in \tilde{\mathfrak{u}}_{-\frac{1}{2}}, y \in \tilde{\mathfrak{s}}_{\frac{1}{2}}$ then by (3)

$$
\langle[\tilde{j} \tilde{s}, u], j y\rangle+\langle[y, \tilde{j} \tilde{s}], j u\rangle+\langle[u, y], j \tilde{j} \tilde{s}\rangle=\langle[u, y], j \tilde{j} \tilde{s}\rangle,
$$

thus $\langle\tilde{j} \tilde{s}, \mathfrak{v}\rangle=0$. Since $\tilde{j} \tilde{s} \in \mathfrak{u}_{0} \oplus \mathfrak{a}$ it follows that $\tilde{j} \tilde{s} \in \mathfrak{z} \oplus \mathfrak{a}$ as claimed.

(iv) if $x \in \tilde{\mathfrak{s}}_{1}, y \in \tilde{\mathfrak{u}}_{\lambda}, \lambda \in\left\{0,1, \frac{1}{2}\right\}$ then

$$
\begin{aligned}
0 & =\langle[\tilde{j} \tilde{s}, x], j y\rangle+\langle[y, \tilde{j} \tilde{s}], j x\rangle+\langle[x, y], j \tilde{j} \tilde{s}\rangle \\
& =(1+\lambda)\langle x, j y\rangle+\langle[x, y], j \tilde{j} \tilde{s}\rangle .
\end{aligned}
$$

Since $\tilde{j} \tilde{s} \in \mathfrak{z} \oplus \mathfrak{a}$ (by (iii)) the last term above vanishes hence $\left\langle\mathfrak{s}_{1}, \tilde{\mathfrak{u}}\right\rangle=0$. By (i) above, $\mathfrak{u}=\tilde{\mathfrak{u}}$, thus the proposition follows.

We showed in $\S 3$ that $u_{0} \oplus \mathfrak{a}$ is abelian and coincides with its own centralizer, hence it is a Cartan subalgebra. It follows from [1] that two Cartan subalgebras of a solvable Lie algebra are conjugated. Hence this fact together with Proposition 4.2 imply

Corollary 4.1. If $(\mathfrak{r}, j,\langle\rangle$,$) and (\mathfrak{r}, \tilde{j},\langle\langle\rangle\rangle$,$) are two split solvable Kähler$ algebras then there exists an automorphism $\alpha$ of $\mathfrak{r}$ such that $\alpha(\mathfrak{u})=\tilde{\mathfrak{u}}$. In particular, in any split solvable Kähler algebra the dimension of the abelian $j$ invariant ideal does not depend on the complex structure.

We are now in a position to prove the main result of this section.

Theorem 4.1. Let $(\mathfrak{r}, j,\langle\rangle$,$) and (\mathfrak{r}, \tilde{j},\langle\langle\rangle\rangle$,$) be two split solvable Kähler$ algebras with $\mathfrak{u}_{0} \oplus \mathfrak{a}=\tilde{\mathfrak{u}}_{0} \oplus \tilde{\mathfrak{a}}$. Then $j s-\tilde{j} \tilde{s} \in \mathfrak{z}$. In particular

$$
\mathfrak{u}_{\lambda}=\tilde{\mathfrak{u}}_{\lambda}, \quad \lambda \in\left\{0, \pm \frac{1}{2}\right\} \quad \text { and } \mathfrak{s}_{\lambda}=\tilde{\mathfrak{s}}_{\lambda}, \quad \lambda \in\left\{0,1, \frac{1}{2}\right\} \text {. }
$$

Proof. By Proposition 4.2, $\tilde{j} \tilde{s} \in \mathfrak{z} \oplus \mathfrak{a}$ hence $\operatorname{ad}_{j \tilde{s}}$ preserves $\mathfrak{s}$. We consider next the decomposition of $\mathfrak{s}$ into eigenspaces of $\operatorname{ad}_{j \grave{s} s}, \mathfrak{s}=\overline{\mathfrak{s}}_{0} \oplus \overline{\mathfrak{s}}_{1} \oplus \overline{\mathfrak{s}}_{\frac{1}{2}} \oplus \overline{\mathfrak{s}}_{-\frac{1}{2}}$. Now $\overline{\mathfrak{s}}_{-\frac{1}{2}}=\mathfrak{s} \cap \tilde{\mathfrak{u}}_{-\frac{1}{2}}$ and by 4.2 (ii), $\tilde{\mathfrak{u}}_{-\frac{1}{2}} \subset \mathfrak{u}$ thus $\overline{\mathfrak{s}}_{-\frac{1}{2}}=0$. Also $\overline{\mathfrak{s}}_{1}=\mathfrak{s} \cap \tilde{\mathfrak{s}}_{1}$ and since $\tilde{\mathfrak{s}}_{1} \subset \mathfrak{s}($ see 4.2$)$ one has $\overline{\mathfrak{s}}_{1}=\tilde{\mathfrak{s}}_{1}$. Thus $\mathfrak{s}=\overline{\mathfrak{s}}_{0} \oplus \tilde{\mathfrak{s}}_{1} \oplus \overline{\mathfrak{s}}_{\frac{1}{2}}$.

Moreover, by the structure theorem of normal $j$-algebras (see 2.2), $\mathrm{ad}_{h}$, $h \in \mathfrak{a}$, is a selfadjoint transformation of $\mathfrak{s}$, hence the previous decomposition is orthogonal' with respect to $\langle$,$\rangle .$ 
We show next that $j \tilde{\mathfrak{s}}_{1}=\overline{\mathfrak{s}}_{0}$ (hence $j \overline{\mathfrak{s}}_{0}=\tilde{\mathfrak{s}}_{1}$ and $\left.j \overline{\mathfrak{s}}_{\frac{1}{2}}=\overline{\mathfrak{s}}_{\frac{1}{2}}\right)$.

Let $x \in \overline{\mathfrak{s}}_{\frac{1}{2}}, y \in \tilde{\mathfrak{s}}_{1}$. Then

$$
0=\langle[\tilde{j} \tilde{s}, x], j y\rangle+\langle[y, \tilde{j} \tilde{s}], j x\rangle=\frac{3}{2}\langle x, j y\rangle
$$

since $\left[\tilde{\mathfrak{s}}_{1}, \overline{\mathfrak{s}}_{\frac{1}{2}}\right]=0$. If $x, y \in \tilde{\mathfrak{s}}_{1}$ then

$$
0=\langle[\tilde{j} \tilde{s}, x], j y\rangle+\langle[y, \tilde{j} \tilde{s}], j x\rangle=2\langle x, j y\rangle
$$

since $\left[\tilde{\mathfrak{s}}_{1}, \tilde{\mathfrak{s}}_{1}\right]=0$. Thus $j \tilde{\mathfrak{s}}_{1}=\overline{\mathfrak{s}}_{0}$ as asserted. Applying now Lemma 3.3 we obtain $-j \tilde{j} \tilde{s}=s+z, z \in \mathfrak{z}$ or $\tilde{j} \tilde{s}-j s \in \mathfrak{z}$ as claimed.

\section{RIGIDITY OF COMPLEX STRUCTURES}

Given a solvable Lie algebra $\mathfrak{r}$ admitting a structure of a solvable Kähler algebra, it will be the purpose of this section to describe all pairs $(j,\langle\rangle$, up to isomorphism (equivalence) turning $(\mathfrak{r}, j,\langle\rangle$,$) into a solvable Kähler$ algebra.

We will say that $(\mathfrak{r}, j,\langle\rangle$,$) and (\tilde{\mathfrak{r}}, \tilde{j},\langle\langle\rangle\rangle$,$) are isomorphic if there exists$ a Lie algebra isomorphism $\sigma$ such that $\tilde{j} \sigma=\sigma j$ (i.e. $\sigma$ is complex linear).

When the Lie algebra isomorphism is complex linear and orthogonal, the triples $(\mathfrak{r}, j,\langle\rangle$,$) and (\tilde{\mathfrak{r}}, \tilde{j},\langle\langle\rangle\rangle$,$) will be said to be equivalent.$

We will consider the cases $\mathfrak{r}$ abelian then $\mathfrak{r}$ a normal $j$-algebra, and finally $\mathfrak{r}$ a split solvable Kähler algebra.

5.1 $\mathfrak{r}$ abelian, $\mathfrak{r}=\mathbf{R}^{2 n}$. If we fix an almost complex structure $j$ in $\mathbf{R}^{2 n}$, (that is, $j^{2}=-\mathrm{id}$ ) then any other almost complex structure $\tilde{j}$ satisfies $\tilde{j}=\tau^{-1} j \tau$ for some $\tau \in G L\left(\mathbf{R}^{2 n}\right)$. If

$$
G L\left(\mathbf{C}^{n}\right)=\left\{T \in G L\left(\mathbf{R}^{2 n}\right): T j=j T\right\}
$$

then $\tau^{-1} G L\left(\mathbf{C}^{n}\right)$ is the set of complex linear isomorphisms of $\left(\mathbf{R}^{2 n}, j\right) \rightarrow$ $\left(\mathbf{R}^{2 n}, \tilde{j}\right)$.

$5.2 \mathfrak{r}=(s, j, \omega)$ a normal $j$-algebra. Given $(\mathfrak{s}, j, \omega)$ a normal $j$-algebra, it follows from Corollary 4.1 that if $(\mathfrak{s}, \tilde{j},\langle\langle\rangle\rangle$,$) is a solvable Kähler algebra$ then it must be a normal $j$-algebra. If the normal $j$-algebra is irreducible, it was proved in [5] that, up to conjugation, there is only one isomorphism class of $j$ on $\mathfrak{s}$. In particular, irreducible homogeneous bounded domains admit only one (up to conjugate) complex structure with a fixed group of automorphisms.

$5.3(\mathfrak{r}, j,\langle\rangle$,$) a split solvable Kähler algebra. Let \mathfrak{r}$ be a split solvable Lie algebra and let $(j,\langle\rangle$,$) and (\tilde{j},\langle\langle\rangle\rangle$,$) be two pairs such that (\mathfrak{r}, j,\langle\rangle$, and $(\mathfrak{r}, \tilde{j},\langle\langle\rangle\rangle$,$) become split solvable Kähler algebras. If \mathfrak{u}_{0} \oplus \mathfrak{a}=\tilde{\mathfrak{u}}_{0} \oplus \tilde{\mathfrak{a}}$ it follows from Theorem 4.1 that $\mathfrak{u}_{\lambda}=\tilde{\mathfrak{u}}_{\lambda}, \lambda \in\{0,1 / 2,-1 / 2\}$ and $\mathfrak{s}_{\lambda}=\tilde{\mathfrak{s}}_{\lambda}$, $\lambda \in\{1,0,1 / 2\}$, in particular $\mathfrak{a}=\tilde{\mathfrak{a}}$.

If $\Lambda=\left\{\varepsilon_{1}, \ldots, \varepsilon_{r}\right\}$ and $\widetilde{\Lambda}=\left\{\tilde{\varepsilon}_{1}, \ldots, \tilde{\varepsilon}_{r}\right\}$ are the sets of fundamental roots of $(s, j,\langle\rangle$,$) and (s, \tilde{j},\langle\langle\rangle\rangle$,$) we may fix a permutation \mu \in S_{r}$ (see [5, Remark 1]) satisfying $\tilde{\varepsilon}_{\mu(i)}=\varepsilon_{i}$. Let $x_{k}, \tilde{x}_{k}$ be the elements in $\mathfrak{n}_{\varepsilon_{k}}, \mathfrak{n}_{\tilde{\varepsilon}_{k}}$ such that $\varepsilon_{i}\left(j x_{k}\right)=\tilde{\varepsilon}_{i}\left(\tilde{j} \tilde{x}_{k}\right)=\delta_{i, k}, k=1, \ldots, r$. Since $j x_{k}$ (resp. $\left.\tilde{j} \tilde{x}_{k}\right)$, $k=1, \ldots, r$, is a basis of $\mathfrak{a}$ (resp. $\tilde{\mathfrak{a}}$ ) it follows that $j x_{k}=\sum a_{i}^{k} \tilde{j} \tilde{x}_{i}$. Then 
$\tilde{\varepsilon}_{\mu(i)} j x_{k}=a_{\mu(i)}^{k}$ and on the other hand $\varepsilon_{i} j x_{k}=\delta_{i, k}$. Thus $a_{\mu(i)}^{k}=\delta_{i, k}$ or equivalently

$$
j x_{k}=\tilde{j} \tilde{x}_{\mu(k)} .
$$

Using the root space decomposition of a split solvable Kähler algebra one easily verifies that

$$
\mathfrak{n}_{\varepsilon_{i}}=\mathfrak{n}_{\tilde{\varepsilon}_{\mu(i)}}, \quad \mathfrak{n}_{\frac{1}{2}\left(\varepsilon_{i} \pm \varepsilon_{j}\right)}=\mathfrak{n}_{\frac{1}{2}\left(\tilde{\varepsilon}_{\mu(i)} \pm \tilde{\varepsilon}_{\mu(j)}\right)}, \quad \mathfrak{n}_{\frac{1}{2} \varepsilon_{i}}=\mathfrak{n}_{\frac{1}{2} \tilde{\varepsilon}_{\mu(i)}}, \quad \mathfrak{u}_{ \pm \frac{1}{2} \varepsilon_{i}}=\mathfrak{u}_{ \pm \frac{1}{2} \tilde{\varepsilon}_{\mu(i)}} .
$$

In particular

$$
\tilde{x}_{\mu(i)}=\mu_{i} x_{i}, \quad \mu_{i} \neq 0 .
$$

Furthermore, it is proved in [5],

$$
\text { if } \frac{1}{2}\left(\varepsilon_{i}+\varepsilon_{j}\right) \text { is a root then } \mu_{i} \mu_{j}>0 .
$$

For any subset $I \subset J=\{1, \ldots, r\}$ we set

$$
\begin{aligned}
\mathfrak{r}_{I}= & \sum_{i \in I}\left[\mathfrak{u}_{-} \frac{\varepsilon_{i}}{2}, \mathfrak{n}_{\frac{\varepsilon_{i}}{2}}\right] \oplus \sum_{i \in I} \mathfrak{u}_{\frac{\varepsilon_{i}}{2}} \oplus \sum_{i \in I} \mathfrak{u}_{-\frac{\varepsilon_{i}}{2}} \oplus \sum_{i \in I} j \mathfrak{n}_{\varepsilon_{i}} \\
& \oplus \sum_{i \in I}\left(\mathfrak{n}_{\varepsilon_{i}} \oplus \mathfrak{n}_{\frac{\varepsilon_{i}}{2}}\right) \oplus \sum_{i, j \in I, i<j} \frac{\mathfrak{n}_{\left(\varepsilon_{i} \pm \varepsilon_{j}\right)}}{2} .
\end{aligned}
$$

If $I^{+}$corresponds to indices $i$ such that $\mu_{i}>0$ and $I^{-}=J-I$ we denote $\mathfrak{r}_{I^{+}}=\mathfrak{r}_{+}, \mathfrak{r}_{I^{-}}=\mathfrak{r}_{-}$. It follows from (10) that if $\frac{1}{2}\left(\varepsilon_{i}+\varepsilon_{j}\right)$ is a root then the indices $i, j$ are in either $I^{+}$or $I^{-}$. Thus $\mathfrak{r}_{+} \cap \mathfrak{r}_{-}=\{0\}$.

Lemma 5.1. $-\tilde{j} j$ is a selfadjoint transformation on $[\mathfrak{u}, \mathfrak{s}] \oplus \mathfrak{s}$, positive definite on $\mathfrak{r}_{+}$and negative definite on $\mathfrak{r}_{-}$.

Proof. Let $u \in \mathfrak{u}_{-\frac{1}{2} \varepsilon_{i}}=\mathfrak{u}_{-\frac{1}{2} \varepsilon_{\mu(i)}}$ and $v \in \mathfrak{u}_{\frac{1}{2} \varepsilon_{i}}=\mathfrak{u}_{\frac{1}{2} \varepsilon_{\mu(i)}}$. From $j u=\left[x_{i}, u\right]=$ $\frac{1}{\mu_{i}}\left[\tilde{x}_{\mu(i)}, u\right]=\frac{1}{\mu_{i}} \tilde{j} u$ and $j v=\mu_{i} \tilde{j} v$ we obtain

$$
\tilde{j} j=-\frac{1}{\mu_{i}} I \text { on } \mathfrak{u}_{-\frac{1}{2} \varepsilon_{i}}, \quad \tilde{j} j=-\mu_{i} I \text { on } \mathfrak{u}_{\frac{1}{2} \varepsilon_{i}} .
$$

If $y \in \mathfrak{n}_{\frac{1}{2}\left(\varepsilon_{i}-\varepsilon_{j}\right)}$, the integrability condition implies that $\left[x_{j}, y\right]=j y,\left[\tilde{x}_{\mu(j)}, y\right]$ $=\tilde{j} y$. Hence on $\sum \mathfrak{n}_{\frac{1}{2}\left(\varepsilon_{i} \pm \varepsilon_{j}\right)}$ we have

$$
\tilde{j} j=-\mu_{j} I \quad \text { on } \mathfrak{n}_{\frac{1}{2}\left(\varepsilon_{i}+\varepsilon_{j}\right)}, \quad \tilde{j} j=-\frac{1}{\mu_{j}} I \quad \text { on } \mathfrak{n}_{\frac{1}{2}\left(\varepsilon_{i}-\varepsilon_{j}\right)} .
$$

Moreover, it follows from (8) and (9) that

$$
\tilde{j} j x_{j}=-\mu_{j} x_{j}, \quad \tilde{j} j=-\frac{1}{\mu_{j}} j x_{j} .
$$

We show next that $\tilde{j} j$ is selfadjoint on $\mathfrak{n}_{\frac{1}{2} \varepsilon_{i}}$.

If $x, y \in \mathfrak{n}_{\frac{1}{2} \varepsilon_{i}}$, then $\langle j \tilde{j} x, y\rangle=-\langle\tilde{j} x, j y\rangle$. From (3) $\left\langle[y, \tilde{j} x], x_{i}\right\rangle=$ $-\langle\tilde{j} x, j y\rangle$ and from $(2)[y, \tilde{j} x]=-[\tilde{j} y, x]$. Hence

$$
\langle j \tilde{j} x, y\rangle=\left\langle[y, \tilde{j} x], x_{i}\right\rangle=-\left\langle[\tilde{j} y, x], x_{i}\right\rangle=\langle x, j \tilde{j} y\rangle
$$

and $j \tilde{j}$ (hence $\tilde{j} j$ ) is selfadjoint. Furthermore, from

$$
[x, \tilde{j} x]=\left\langle[x, \tilde{j} x], x_{i}\right\rangle \frac{x_{i}}{\omega\left(x_{i}\right)}=\left\langle\left\langle[x, \tilde{j} x], \tilde{x}_{\mu(i)}\right\rangle\right\rangle \frac{\tilde{x}_{\mu(i)}}{\tilde{\omega}\left(\tilde{x}_{\mu(i)}\right)}
$$


we obtain

$$
\left\langle[x, \tilde{j} x], x_{i}\right\rangle=\frac{\omega\left(x_{i}\right)}{\tilde{\omega}\left(\tilde{x}_{\mu(i)}\right)} \mu_{i}\left\langle\left\langle[x, \tilde{j} x], \tilde{x}_{\mu(i)}\right\rangle\right\rangle .
$$

Thus

$$
\langle j \tilde{j} x, x\rangle=-\langle\tilde{j} x, j x\rangle=\left\langle[x, \tilde{j} x], x_{i}\right\rangle=-\frac{\omega\left(x_{i}\right)}{\tilde{\omega}\left(\tilde{x}_{\mu(i)}\right)} \mu_{i}\langle\langle\tilde{j} x, \tilde{j} x\rangle\rangle
$$

and the sign of $j \tilde{j}$ on $\mathfrak{n}_{\frac{1}{2} \varepsilon_{i}}$ depends on $\mu_{i}$ as claimed.

According to Proposition 3.1, $\mathfrak{u}_{0}=\mathfrak{z} \oplus \sum\left[\mathfrak{u}_{-\frac{1}{2} \varepsilon_{i}}, \mathfrak{n}_{\frac{1}{2} \varepsilon_{i}}\right]$. Thus if $u_{0}=[u, x] \in$ $\left[\mathfrak{u}_{-\frac{1}{2} \varepsilon_{i}}, \mathfrak{n}_{\frac{1}{2} \varepsilon_{i}}\right]$ and $w \in \mathfrak{u}_{0}$ one has $\tilde{j} j[u, x]=[u, \tilde{j} j x]$, hence

$$
\langle\tilde{j} j[u, x], j w\rangle=\langle[u, \tilde{j} j x], j w\rangle=\langle[w, \tilde{j} j x], j u\rangle .
$$

Again, from $\tilde{j}[w, j x]=[\tilde{j} w, j x]+[w, \tilde{j} j x]+\tilde{j}[\tilde{j} w, \tilde{j} j x]$ one obtains

$$
\begin{gathered}
{[w, j x]=[\tilde{j} w, \tilde{j} j x] \quad\left(\text { both are in } \mathfrak{u}_{\frac{1}{2} \varepsilon_{i}}\right),} \\
{[\tilde{j} w, j x]=-[w, \tilde{j} j x] \quad\left(\text { both are in } \mathfrak{u}_{-\frac{1}{2} \varepsilon_{i}}\right) .}
\end{gathered}
$$

If we substitute the last equality in $\langle[w, \tilde{j} j x], j u\rangle$ above, we obtain

$$
\begin{aligned}
\langle\tilde{j} j[u, x], j w\rangle & =-\langle[\tilde{j} w, j x], j u\rangle=-\langle[u, j x], j \tilde{j} w\rangle \\
& =-\langle j[u, x], j \tilde{j} w\rangle=\langle[u, x], \tilde{j} j j w\rangle .
\end{aligned}
$$

Thus $\tilde{j} j$ is selfadjoint on $\sum\left[\mathfrak{u}_{-\frac{1}{2} \varepsilon_{i}}, \mathfrak{n}_{\frac{1}{2} \varepsilon_{i}}\right]$. Note that the eigenvalues of $\tilde{j} j$ on $\left[\mathfrak{u}_{-\frac{1}{2} \varepsilon_{i}}, \mathfrak{n}_{\frac{1}{2} \varepsilon_{i}}\right]$ coincide with the eigenvalues on $\mathfrak{n}_{\frac{1}{2} \varepsilon_{i}}$ since $\tilde{j} j[u, x]=[u, \tilde{j} j x]$. Hence the lemma follows.

Remark 3. It is not true in general that given $\left(\mathbf{C}^{n}, j\right)$ and $\left(\mathbf{C}^{n}, \tilde{j}\right)$, the transformation $\tilde{j} j$ is selfadjoint. For example, if $n=1, j=\left(\begin{array}{cc}0 & -1 \\ 1 & 0\end{array}\right)$ and $T=\left(\begin{array}{ll}1 & a \\ 0 & b\end{array}\right)$, $b \neq 0$, then considering $\tilde{j}=T j T^{-1}=\left(\begin{array}{c}a-\frac{a^{2}+1}{b} \\ b-a+1\end{array}\right)$ one obtains $\tilde{j} j=\left(\begin{array}{c}-\frac{a^{2}+1}{b}-a \\ -(a+1)-b\end{array}\right)$.

Remark 4. Let $j$ and $\tilde{j}$ be two endomorphisms of a vector space $V$ such that $j^{2}=\tilde{j}^{2}=-I$. Assume $-\tilde{j} j$ is selfadjoint and decompose,

$$
V=V_{+} \oplus V_{-}, \quad V_{+}=\sum_{\lambda>0} V_{\lambda}, \quad V_{-}=\sum_{\lambda<0} V_{\lambda}
$$

where $V_{\lambda}$ stands for an eigenspace of $-\tilde{j} j$. Then $V_{+}$and $V_{-}$are $j$ (resp. $\tilde{j}$ ) invariant. (This is clearly so since from $\tilde{j} j x=\lambda x$ one obtains $\tilde{j} x=-\frac{1}{\lambda} j x$ hence $\tilde{j} j(j x)=\frac{1}{\lambda} j x$ and $\tilde{j} j(\tilde{j} x)=\frac{1}{\lambda} \tilde{j} x$.) Furthermore on $V_{+}$one has $\tilde{j}$ equivalent to $-j$. In fact, if $x \in V_{\lambda}, \lambda>0$,

$$
\tilde{j}(-\tilde{j} j)^{\frac{1}{2}} x=\lambda^{\frac{1}{2}} \tilde{j} x=\frac{1}{\lambda^{\frac{1}{2}}} j x, \quad(-\tilde{j} j)^{\frac{1}{2}}(j x)=\frac{1}{\lambda^{\frac{1}{2}}} j x
$$

and similarly $\tilde{j}$ is equivalent to $-j$ on $V_{-}$.

Theorem 5.1. Let $(\mathfrak{r}, j,\langle\rangle$,$) and (\mathfrak{r}, \tilde{j},\langle\langle\rangle\rangle$,$) be two split solvable Kähler$ algebras. Then

(i) $\mathfrak{r}$ splits into a sum of $j$ and $\tilde{j}$ invariant ideals, $\mathfrak{r}=\mathfrak{z} \oplus \mathfrak{r}_{+} \oplus \mathfrak{r}_{-}$. 
(ii) There exist a complex linear automorphism $\alpha_{+}: \mathfrak{z} \oplus \mathfrak{r}_{+} \rightarrow \mathfrak{z} \oplus \mathfrak{r}_{+}$and a conjugate linear automorphism $\alpha_{-}: \mathfrak{z} \oplus \mathfrak{r}_{-} \rightarrow \mathfrak{z} \oplus \mathfrak{r}_{-}$.

Proof. If $\sigma$ is an automorphism of $\mathfrak{r}$ such that $\sigma\left(\mathfrak{u}_{0} \oplus \mathfrak{a}\right)=\tilde{\mathfrak{u}}_{0} \oplus \tilde{\mathfrak{a}}$ then $(\mathfrak{r}, \tilde{j},\langle\langle\rangle\rangle$,$) is equivalent to its pullback via \sigma$. Thus we may assume $\mathfrak{u}_{0} \oplus \mathfrak{a}=\tilde{\mathfrak{u}}_{0} \oplus \tilde{\mathfrak{a}}$.

Setting $\mathfrak{r}_{+}$and $\mathfrak{r}_{-}$as in 11 , it follows from Remark 4 that both are $j$ and $\tilde{j}$ invariant. Also, nonzero brackets may appear only when considering elements of $\mathfrak{r}_{+}$or $\mathfrak{r}_{-}$hence $\mathfrak{r}_{+}$and $\mathfrak{r}_{-}$are ideals. Since $\mathfrak{z}$ is also $j$ and $\tilde{j}$ invariant (see 2.1), (i) follows.

To prove (ii) there is no loss of generality in assuming $\mathfrak{z} \oplus \mathfrak{r}_{+}=\mathfrak{r}$ (since we may change $j$ by $-j$ in $\mathfrak{r}_{-}$. ) We will also assume that $\mathfrak{z}=0$ and show that there exists an automorphism $\alpha_{+}: \mathfrak{r} \rightarrow \mathfrak{r}$ such that $\tilde{j} \alpha_{+}=\alpha_{+} j$. For that purpose define $\alpha_{+}$as follows

$$
\begin{aligned}
\alpha_{+} u & =(-\tilde{j} j)^{\frac{1}{2}} u, & & u \in \mathfrak{u} ; \\
\alpha_{+} h & =h, & & h \in \mathfrak{a} ; \\
\alpha_{+} x_{i} & =\mu_{i} x_{i}, & & x_{i} \in \mathfrak{n}_{\varepsilon_{i}} ; \\
\alpha_{+} x & =\left(\frac{\mu_{i}}{\mu_{j}}\right)^{\frac{1}{2}} x, & & x \in \mathfrak{n}_{\frac{1}{2}\left(\varepsilon_{i}-\varepsilon_{j}\right)} ; \\
\alpha_{+} x & =\left(\mu_{i} \mu_{j}\right)^{\frac{1}{2}} x, & & x \in \mathfrak{n}_{\frac{1}{2}\left(\varepsilon_{i}+\varepsilon_{j}\right)} ; \\
\alpha_{+} x & =\mu_{i}^{\frac{1}{2}}(-\tilde{j} j)^{\frac{1}{2}} x, & & x \in \mathfrak{n}_{\frac{1}{2} \varepsilon_{i}} .
\end{aligned}
$$

It is not hard to check using Remark 4 and equations (8), (9), (13) that $\tilde{j} \alpha_{+}=\alpha_{+} j$. We show next that $\alpha_{+}$is an automorphism.

- If $u \in \mathfrak{u}_{\alpha}, h \in \mathfrak{a}, \alpha_{+}[u, h]=\alpha(h) \alpha_{+}(u)=\left[h, \alpha_{+} u\right]$;

- if $u \in \mathfrak{u}_{\frac{1}{2} \varepsilon_{j}}, x \in \mathfrak{n}_{\frac{1}{2}\left(\varepsilon_{i}-\varepsilon_{j}\right)}$ then using (12) $\alpha_{+}[u, x]=\left(\mu_{i}\right)^{\frac{1}{2}}[u, x]$ and $\left[\alpha_{+} u, \alpha_{+} x\right]=\left[\mu_{j}^{\frac{1}{2}} u,\left(\frac{\mu_{i}}{\mu_{j}}\right)^{\frac{1}{2}} x\right]$;

- if $u \in \mathfrak{u}_{-\frac{1}{2} \varepsilon_{j}}, x \in \mathfrak{n}_{\frac{1}{2}\left(\varepsilon_{i}+\varepsilon_{j}\right)}$ then (3) implies $[u, x]=[j u, j x]$ hence $\alpha_{+}[u, x]=\alpha_{+}[j u, j x]=\left[\alpha_{+} j u, \alpha_{+} j x\right]=\left[\tilde{j} \alpha_{+} u, \tilde{j} \alpha_{+} x\right]=\left[\alpha_{+} u, \alpha_{+} x\right]$;

- if $u \in \mathfrak{u}_{-\frac{1}{2} \varepsilon_{j}}, x \in \mathfrak{n}_{\frac{1}{2} \varepsilon_{j}}$ then $\alpha_{+}[u, x]=\left[u,(-\tilde{j} j)^{\frac{1}{2}} x\right]=\frac{1}{\mu_{i}^{1 / 2}}\left[u, \alpha_{+} x\right]=$ $\left[\alpha_{+} u, \alpha_{+} x\right]$ (since $\left.\tilde{j} j u=-\frac{1}{\mu_{i}} u\right)$;

- if $u_{0}=[u, x], u \in \mathfrak{u}_{-\frac{1}{2} \varepsilon_{i}}, x \in \mathfrak{n}_{\frac{1}{2} \varepsilon_{i}}$ and $y \in \mathfrak{n}_{\frac{1}{2} \varepsilon_{i}}$ then $\alpha_{+}[[u, x], y]=$ $\left(\mu_{i}\right)^{\frac{1}{2}}[[u, x] y]$ and

$$
\left[\alpha_{+}[u, x], \alpha_{+} y\right]=\mu_{i}^{\frac{1}{2}}\left[(-\tilde{j} j)^{\frac{1}{2}}[u, x],(-\tilde{j} j)^{\frac{1}{2}} y\right]=\alpha_{+}[[u, x], y]
$$

where the last equality follows from

$$
\begin{aligned}
& \left\langle(-\tilde{j} j)^{\frac{1}{2}} u_{0},(-\tilde{j} j)^{\frac{1}{2}} x, j v\right\rangle=\left\langle\left[v,(-\tilde{j} j)^{\frac{1}{2}} x\right], j(-\tilde{j} j)^{\frac{1}{2}} u_{0}\right\rangle \\
& \quad=\left\langle(-\tilde{j} j)^{\frac{1}{2}}[v, x], j(-\tilde{j} j)^{\frac{1}{2}} u_{0}\right\rangle=\left\langle[v, x],(-\tilde{j} j)^{\frac{1}{2}} j(-\tilde{j} j)^{\frac{1}{2}} u_{0}\right\rangle \\
& \quad=\left\langle[v, x], \tilde{j}(-\tilde{j} j) u_{0}\right\rangle=\left\langle[v, x], j u_{0}\right\rangle=\left\langle\left[u_{0}, x\right], j v\right\rangle .
\end{aligned}
$$

It was proved in [5] that $\alpha_{+}$is an automorphism when restricted to $s$ thus our assertion follows. If $\mathfrak{z} \neq 0$ we define $\alpha_{+}$when restricted to the center, as any complex linear transformation (compare $\S 5.1$ ).

From the previous theorem the next corollary follows immediately. 
Corollary 5.1. Let $(\mathfrak{r}, j,\langle\rangle$,$) and (\tilde{\mathfrak{r}}, \tilde{j},\langle\langle\rangle\rangle$,$) be split solvable Kähler$ algebras with $(\mathfrak{r}, j,\langle\rangle$,$) irreducible and \mathfrak{r}$ isomorphic (as Lie algebras) to $\tilde{\mathfrak{r}}$. Then either $(\mathfrak{r}, j,\langle\rangle$,$) is isomorphic to (\tilde{\mathfrak{r}}, \tilde{j},\langle\langle\rangle\rangle$,$) or it is isomorphic to$ $(\tilde{\mathfrak{r}},-\tilde{j},\langle\langle\rangle$,$) .$

\section{Proof of Theorem 6.1}

As we mentioned in the Introduction, the proof of Theorem 6.1 rests on modifications of solvable Lie algebras. Many authors have previously used this notion (see for example [1, 4, 7]). We essentially follow [7].

Let $(\mathfrak{r}, j,\langle\rangle$,$) be a solvable Kähler algebra, let (R, j,\langle\rangle$,$) be the cor-$ responding simply-connected Kähler solvmanifold and let $G$ be the group of automorphisms of $(R, j,\langle\rangle$,$) with isotropy subgroup K$ at the identity in $R$. We denote by $\mathfrak{g}$ and $\mathfrak{k}$ the Lie algebras of $G$ and $K$ respectively.

Let $\phi: \mathfrak{r} \rightarrow \mathfrak{k}$ be a linear map satisfying

1. $[\phi(\mathfrak{r}), \mathfrak{r}] \subset \mathfrak{r}$,

2. $(I+\phi) \mathfrak{r}$ is a solvable subalgebra of $\mathfrak{g}$,

3. $[\phi(\mathfrak{r}),(I+\phi) \mathfrak{r}] \subset(I+\phi) \mathfrak{r}$.

The subalgebra $(I+\phi) \mathfrak{r}$ is a normal modification of $\mathfrak{r}$ and the map $\phi$ is a normal modification map. If $R^{\phi}$ denote the connected subgroup of $G$ with Lie algebra $(I+\phi) \mathfrak{r}$ then $R^{\phi}$ is said to be a normal modification of $R$.

According to $[7,(2.10)]$ and because $R$ is simply connected, $R^{\phi}$ acts simply transitively on $R$. Thus $(I+\phi) \mathfrak{r}$ inherits a structure of a solvable Kähler algebra; furthermore, if $\mathfrak{r}$ is irreducible, its normal modification $(I+\phi) \mathfrak{r}$ is also irreducible. If $\left(j^{\phi},\langle,\rangle_{\phi}\right)$ denote the pullback to $R^{\phi}$ of the Kähler structure $(j,\langle\rangle$,$) on R$, it is clear that $G$ can be identified with the group of automorphisms of $\left(R^{\phi},\left(j^{\phi},\langle,\rangle_{\phi}\right)\right.$.

According to Dorfmeister [4], every solvable Kähler algebra can be modified to obtain a split solvable Kähler algebra. This result together with Theorem 5.1 imply

Theorem 6.1. Let $(M, j,\langle\rangle$,$) be an irreducible Kähler solvmanifold with$ $M$ simply connected and $G$ the group of automorphisms of $M$. Then any other Kähler structure $(\tilde{j},\langle\langle\rangle\rangle$,$) on M$ with group of automorphisms $G$ is isomorphic to $(j,\langle\rangle$,$) .$

Proof. Since $M$ is simply connected, it can be identified with a solvable Lie group $R$. Thus we are reduced to consider the solvable Kähler algebras $(\mathfrak{r}, j,\langle\rangle$,$) , and (\mathfrak{r}, \tilde{j},\langle\langle\rangle\rangle$,$) with (r, j,\langle\rangle$,$) irreducible. Furthermore,$ after a series of modifications, we end with $\left(\mathfrak{r}_{1}, j,\langle\rangle,\right)$ and $\left(\mathfrak{r}_{1}, \tilde{j},\langle\langle\rangle\rangle,\right), \mathfrak{r}_{1}$ split solvable [5] and $(\mathfrak{r}, j,\langle\rangle$,$) irreducible. By Theorem 5.1$ there exists a complex linear or conjugate linear isomorphism from $\left(\mathfrak{r}_{1}, j,\langle\rangle,\right)$ onto $\left(\mathfrak{r}_{1}, \tilde{j},\langle\langle\rangle\rangle,\right)$ which can be lifted to a biholomorphic map from $(M, j,\langle\rangle$, onto $(M, \pm \tilde{j},\langle\langle\rangle$,$) .$

\section{REFERENCES}

1. D. V. Alekseevskii, Homogeneous riemannian spaces of negative curvature, Math. USSR-Sb. 25 (1975), 87-109.

2. J. E. D'Atri, The curvature of homogeneous Siegel domains, J. Differential Geom. 15 (1980), $61-70$. 
3. Holomorphic sectional curvatures of bounded homogeneous domains and related questions, Trans. Amer. Math. Soc. 256 (1979), 405-413.

4. J. Dorfmeister, Homogeneous Kähler manifolds admitting a transitive solvable group of automorphisms, Ann. Sci. Ecole Norm. Sup. 18 (1985), 143-188.

5. I. Dotti Miatello, Complex structures on normal j-algebras, J. Pure Appl. Algebra 73 (1991), 247-256.

9. S. G. Gindikin, I. I. Pyatetskii-Shapiro, and E. B. Vinberg, Homogeneous Kähler manifolds, Geometry of Homogeneous Bounded Domains, C.I.M.E., 1967, pp. 1-88.

7. C. S. Gordon and E. N. Wilson, Isometry groups of riemannian solvmanifolds, Trans. Amer. Math. Soc. 307 (1988), 245-269.

8. I. I. Pyatetskii-Shapiro, Automorphic functions and the geometry of classical domains, Gordon and Breach, New York, 1969.

famaf, Universidad Nacional de Córdoba, Valparaiso y Rogelio Martinez, 5000 Córdoba, Argentina 